\title{
High Efficacy and Safety of Flat-Dose Ribavirin Plus Sofosbuvir/Daclatasvir in Genotype 3 Cirrhotic Patients
}

\begin{abstract}
Adriano Pellicelli ${ }^{1}$, Vincenzo Messina ${ }^{2}$, Valerio Giannelli ${ }^{1}$, Marco Distefano ${ }^{3}$, Valeria Pace Palitti ${ }^{4}$, Pascal Vignally ${ }^{5}$, Pierluigi Tarquini ${ }^{6}$, Antonio Izzi ${ }^{7}$, Alessandra Moretti ${ }^{8}$, Sergio Babudieri ${ }^{9}$, Serena Dell'Isola ${ }^{10}$, Massimo Marignani ${ }^{11}$, Gaetano Scifo $^{3}$, Vincenzo lovinella ${ }^{12}$, Giuseppe Cariti ${ }^{13}$, Maurizio Pompili ${ }^{14}$, Francesco Di Candilo ${ }^{15}$, Luca Fontanella ${ }^{16}$, Giuseppe M. Ettorre $^{17}$, Giovanni Vennarecci ${ }^{17}$, Antonio Massimo Ippolito ${ }^{18}$, and Giorgio Barbarini ${ }^{19}$, for the Working Group CLEO

${ }^{1}$ Liver and Transplant Unit, San Camillo Forlanini Hospital, Rome, ${ }^{2}$ Department of Infectious Disease, Sant'Anna and San Sebastiano Hospital, Caserta, ${ }^{3}$ Liver Unit, Azienda Umberto I Hospital, Siracusa, ${ }^{4}$ Liver Unit, Department of Medicine, ASL Pescara, Pescara, ${ }^{5}$ Department of Emergency, Galliera Hospital, Genoa, ${ }^{6}$ Department of Infectious Disease, Giuseppe Mazzini Hospital, Teramo, ${ }^{7}$ Department of Infectious Disease and Emergency Infectious Disease, Cotugno Hospital, Napoli, ${ }^{8}$ Department of Gastroenterology, San Filippo Neri Hospital, Rome, ${ }^{9}$ Department of Infectious Disease, University of Sassari, Sassari, ${ }^{10}$ Department of Infectious Disease, Belcolle Hospital, Viterbo, ${ }^{11}$ Digestive and Liver Disease Unit, Sant'Andrea Hospital, Rome, ${ }^{12}$ Department of Internal Medicine, San Paolo Hospital, Naples, ${ }^{13}$ Infectious Disease, Department of Medical Science, University of Turin, Turin, ${ }^{14}$ Department of Internal Medicine, Catholic University, Rome, ${ }^{15}$ Department of Infectious Disease, Perugia Hospital, Perugia, ${ }^{16}$ Center for Liver Disease, Fatebenefratelli Hospital, Napoli, ${ }^{17}$ Division of General Surgery and Liver Transplantation, San Camillo Forlanini Hospital, Rome, ${ }^{18}$ Division of Gastroenterology, Casa Sollievo Sofferenza Hospital IRCCS, San Giovanni Rotondo, and ${ }^{19}$ Department of Infectious Disease, IRCCS San Matteo, Pavia, Italy
\end{abstract}

Background/Aims: Patients with genotype 3 hepatitis $C$ virus (G3-HCV) cirrhosis are very difficult to treat compared to patients with other HCV genotypes. The optimal treatment duration and drug regimen associated with ribavirin (RBV) remain unclear. To evaluate the efficacy and safety of daclatasvir (DCV)/sofosbuvir (SOF) plus a flat dose of $800 \mathrm{mg}$ RBV (flat dose) compared to DCV/SOF without RBV or DCV/ SOF plus an RBV dose based on body weight (weight-based) in G3-HCV patients with compensated or decompensated cirrhosis. Methods: We analyzed data for 233 G3 cirrhotic patients. Of these, 70 (30\%), 87(37\%) and 76 (33\%) received SOF/DCV, SOF/DCV/RBV flat dose, and SOF/DCV/ RBV weight-based dose, respectively. Treatment duration was 24 weeks. Sustained virological response (SVR) was evaluated at week 12 posttreatment (SVR12). Results: Overall, SVR12 was achieved in 220 out of 233 patients (94.4\%). The SVR12 rate was lower in the DCV/SOF group than in the DCV/SOF/RBV flat-dose group and the DCV/ SOF/RBV weight-based group (87.1\% vs $97.7 \%$ and $97.4 \%$, respectively, $p=0.007)$. A higher incidence of anemia occurred in the DCV/SOF/RBV weight-based group compared to those in the other two groups $(p<0.007)$. Conclusions: We found that the DCV/SOF/RBV flat-dose regimen is an effective treatment in terms of efficacy and safety in patients with G3-HCV compensated or decompensated cir- rhosis. Therefore, antiviral regimens without RBV should be restricted only to naïve patients with G3-HCV compensated cirrhosis who have a clear contraindication for RBV. (Gut Liver 2020;14:357-367)

Key Words: Daclatasvir; Cirrhosis, liver; Genotypes 3; Hepatitis C; Drug therapy; Ribavirin

\section{INTRODUCTION}

Patients infected with genotype 3 hepatitis C virus (G3-HCV) that have compensated and decompensated liver cirrhosis are very difficult to treat. Although direct-acting antiviral agents (DAAs) have improved the sustained virological response (SVR) in all HCV genotypes, patients with G3-HCV-related cirrhosis, whether treatment-experienced or treatment-naïve, remain a therapeutic challenge. The suboptimal virological response in patients with G3-HCV is probably related to the high degree of viral steatosis and the relatively rapid progression to liver fibrosis. ${ }^{1,2}$ Several cohorts and real-life studies have shown that SVR has improved with the combination of daclatasvir (DCV) plus sofosbuvir (SOF), with or without ribavirin (RBV). Moreover, a 12-week or 16-week treatment duration was shown to be suboptimal in patients with cirrhosis. ${ }^{3}$ A 24-week treatment duration is indicated in this population of patients with this antiviral

Correspondence to: Adriano Pellicelli

Liver Unit, Department of Liver Transplantation, San Camillo Forlanini Hospital, Rome 00152, Italy

Tel: +39-0658703472, Fax: +39-0658704667, E-mail: adriano.pellicelli@uniroma1.it

Received on June 11, 2018. Revised on December 12, 2018. Accepted on December 21, 2018. Published online August 14, 2019. pISSN 1976-2283 eISSN 2005-1212 https://doi.org/10.5009/gnl18269

(a) This is an Open Access article distributed under the terms of the Creative Commons Attribution Non-Commercial License (http://creativecommons.org/licenses/by-nc/4.0) which permits unrestricted non-commercial use, distribution, and reproduction in any medium, provided the original work is properly cited. 
regimen. The use of a full dose of RBV in addition to DCV/SOF in patients with G3-HCV cirrhosis could lead to lower adherence and a higher rate of adverse events (AEs). In the present study, we explored SVR and the adherence to a 24-week treatment of DCV/SOF plus a flat dose (800 mg) of RBV in a real, difficult-totreat population of patients with G3-HCV and cirrhosis classified as Child-Pugh-Turcotte A or B (CPT-A or CPT-B). We compared SVR12 between patients treated with DCV/SOF without RBV, DCV/SOF plus flat dose RBV and DCV/SOF plus weightbased RBV.

\section{MATERIALS AND METHODS}

This retrospective multicenter non-randomized open label study included 253 patients with G3-HCV compensated or decompensated cirrhosis. Patients received regimens of $60 \mathrm{mg}$ DCV plus $400 \mathrm{mg}$ SOF, without RBV or with RBV at a flat dose of $800 \mathrm{mg}$ or RBV dose based on body weight, daily for 24 weeks. The choice to use RBV or not and the dosage of RBV was at the discretion of the treating physician on the basis of characteristics of the patient. Patients were enrolled from February 2015 through April 2016 in 12 clinical settings within the Club Epatologi Ospedalieri (CLEO) Group. A total of 233 patients were treated, and their data were retrieved from a centralized database at San Camillo Forlanini Hospital in Rome, Italy. Patient eligibility criteria were: age $>18$ years, G3-HCV infection, compensated CPT-A or decompensated CPT-B cirrhosis, with or without prior treatment experience (treatmentexperienced or treatment-naïve, respectively). The main exclusion criteria were: human immunodeficiency virus infection, hepatitis B infection, active hepatocellular carcinoma (HCC), history of organ transplant, severe psychiatric disorders, or treatment with drugs that showed an interaction with an antiviral DAA-based treatment. Patients were divided into three groups, according to the RBV treatment schedule utilized: group I received DCV/SOF without RBV; group II DCV/SOF with a flat dose (800 mg) of RBV; and group III DCV/SOF with an RBV dose based on body weight. We included patients that were either treatment-experienced or treatment-naïve; prior treatments included peginterferon (PegIFN)/RBV, PegIFN/telaprevir, or SOF/RBV. The presence of cirrhosis was documented with a liver biopsy analysis or with a liver stiffness measured by transient elastography value $>12.5 \mathrm{kPa}$ (Fibroscan; Echosens, Paris France), performed within 6 months before starting therapy. Before starting antiviral treatment, to rule out focal liver lesions, all patients underwent an abdominal ultrasound or computed tomography (CT) and magnetic resonance imaging (MRI) when indicated. Patients with a history of HCC treatment underwent CT or MRI to exclude recurrent or residual HCC. At the end of therapy, and 24 weeks posttreatment, patients underwent another abdominal ultrasound, CT, or MRI examination. This study was conducted in accordance with the
Declaration of Helsinki and it was reviewed and approved by local institutional review board.

\section{Study assessment}

HCV-RNA levels were assessed with the Abbott Real Time HCV viral load assay. The lower limit of quantification (LLOQ) was $12 \mathrm{IU} / \mathrm{mL}$ for a $0.5 \mathrm{~mL}$ sample volume (Abbott Laboratories, Abbott Park, IL, USA). HCV RNA levels were assessed at baseline, during treatment, at 4, 8, 12, and 24 weeks, and posttreatment, at 4 and 12 weeks. HCV genotypes were determined with the Versant HCV genotype 2.0 assay. HCV genotyping was repeated when the results were older than 5 years. Virologic failure was defined as: (1) a virologic breakthrough (confirmed HCV RNA >1 log, or an increase in HCV RNA from the nadir, or confirmed HCV RNA >LLOQ after a previous on-treatment measurement <LLOQ); (2) an incomplete virological response (confirmed HCV-RNA > LLOQ at week 8), or (3) a relapse (confirmed HCV RNA > LLOQ following an undetectable measurement at the end of treatment). Host interleukin 28B (re12979860) was determined and reported when available (Monogram Biosciences, South San Francisco, CA, USA). In patients that lacked a virological response to treatment, blood samples were analyzed for resistance-associated variants (RAVs) in NS5A and NS5B proteins with populationbased sequencing. The presence of RAVs was assessed according to Lontok et al. ${ }^{4}$ Safety assessments were based on AEs and severe adverse events (SAEs), determined with clinical and laboratory tests. Anemia was diagnosed when the hemoglobin level was $<10 \mathrm{~g} / \mathrm{dL}$.

\section{Study end-point}

The primary endpoint was the proportion of patients with HCV RNA <LLOQ at posttreatment week 12 (SVR12). The secondary endpoints included the rates of AEs and treatment discontinuations, due to SAEs.

\section{Statistical analysis}

The statistical analysis of data was done using SPSS program version 20.0 (IBM Corp., Armonk, NY, USA). Categorical data were presented as numbers (percentage). Value are expressed as the mean \pm standard deviation (SD). Differences in baseline characteristics between the three groups of patients were compared with the chi-square and/or two-tailed Fisher exact test for dichotomous variables. For data with normal distribution descriptive statistics were used to calculate mean $\pm S D$; one-way analysis of variance test was used to compare the results between the three groups.

Parameters of viral response were compared among the various groups with the two-tailed Fisher exact test. To identify predictive factors for SVR12, univariate and multivariate logistic regression analyses were performed, with odds ratios (ORs) and 95\% confidence intervals (CIs). Independent risk factors were 
selected for testing in multivariate analyses based on their significance in a prior univariate analysis.

Two-sided p-values $<0.05$ were considered statistically significant. The sample size for each group in the study was determined to obtain a statistical power of around 90\% with an alpha risk of $0.05 \%$. We calculated that a minimum of 204 patients was required for the entire study, or at least 68 patients per group.

\section{RESULTS}

\section{Patient baseline characteristics}

Of 253 consecutive patients treated with DCV/SOF with or without RBV for 24 weeks, 11 patients were lost to follow-up, and nine patients withdrew for undocumented reasons. A total of 233 patients were enrolled in the study. Seventy patients received DCV/SOF (group I); 87 received DCV/SOF plus a flat dose of $800 \mathrm{mg}$ RBV (group II); and 76 received DCV/SOF plus a weight-based dose of RBV (group III). The cirrhosis diagnosis was established according histological criteria in 72 patients, a liver stiffness measurement by transient elastography value $>12.5 \mathrm{kPa}$ in 152 patients, or a combination of clinical and ultrasound imaging data (irregular margins on an ultrasound, or the presence of ascites or esophageal varices) in 65 patients. A total of 197 patients (84.1\%) were male. Cirrhosis was classified as compensated CPT-A in 205 patients (88\%), CPT-B in 27 patients (11.6\%), and CPT-C in only one patient (0.4\%). Previously treated HCC was reported in six patients $(8.7 \%)$ of group I, three patients (3.5\%) of group II, and four patients (5.3\%) of group III. Diabetes, arterial hypertension (HTA), and the presence of cryoglobulinemia were equally distributed among the three groups of patients. Hypercholesterolemia was noted to be more frequent in group I. A total of 124 patients (53.2\%) were treatment-naïve, and 109 (46.8\%) were treatment-experienced. In particular in experienced patients, 52 (22.3\%) had relapsed after PegIFN/ RBV, 49 (21\%) showed no response to PegIFN/RBV, five (2.1\%) showed no response to Peg/IFN/RBV/telaprevir, and three (1.3\%) had relapsed after SOF/RBV. Baseline characteristics of the three groups are reported in Table 1.

\section{Virological response}

A summary of the virological response is reported in Table 2. At the end of treatment a total of 232 patients (99.6\%) had HCV RNA levels <LLOQ. In groups I, II, and III, virological responses were achieved at end of treatment in, respectively, 98.6\%, 100\%, and $100 \%$ of patients. Overall, SVR12 was achieved in 220 out of 233 patients (94.4\%). In group I, 61 out of 70 patients (87.1\%) achieved SVR12; one patient (1.6\%) had a breakthrough, and eight patients relapsed after the end of treatment. In group II, SVR12 was achieved in 85 out of 87 patients (97.7\%); two patients (2.3\%) relapsed, and one patient (1.1\%) dropped out of treatment due to a recurrence of HCC. In group III, SVR12 was achieved in 74 out of 76 patients (97.4\%), and two patients (2.6\%) relapsed. Although not statistically significant, in group I, a larger proportion of treatment-naïve patients achieved SVR12 compared to the treatment-experienced patients (91.5\% vs 76.5\%; OR, 2.99; 95\% CI, 0.6 to $15.4 ; \mathrm{p}=0.1$ ). This difference was not observed in the other groups (group II: naïve vs experienced, 97\% vs 98.1\%; group III: naïve vs experienced, $100 \%$ vs $93.8 \%$ ). Overall, SVR12 was achieved by $96.1 \%$ of patients with CPT-A and $82.1 \%$ of patients with CPT-B/C (OR, 5.3; 95\% CI, 1.3 to 20; $<<0.002$ ). CPT-A patients treated with flat or weight dose RBV had higher SVR12 compared to CPTA patients treated without RBV. Furthermore, CPT-A patients treated with flat dose RBV had similar SVR12 and fewer AEs compared to patients treated with weight dose RBV. In CPT$\mathrm{B} / \mathrm{C}$ patients, weight dose RBV showed more efficacy in terms of SVR12 but had higher AEs compared to patients treated with flat dose and without RBV. All the results are reported in Table 3.

In CPT-A naïve patients, SVR12 was similar between the three groups (group I 97.1\%, group II 100\%, and group III 100\%). SVR 12 was lower in group I CPT-A experienced patients compared to group II $(77.3 \%$ vs $100 \%, \mathrm{p}<0.02)$ and group III $(77.3 \%$ vs 93.5\%, p<0.08). Among patients with CPT-B, 78.6\%, 77.8\%, and 100\% achieved SVR12 in groups I, II, and III, respectively. In group II CPT-B patients, only 25\% (2/8 patients) were naïve and one patient relapsed from a previous treatment with SOF plus RBV, while 60\% (three patients) of patients of group III were naïve.

A total of 13 patients showed no virological response; five with CPT-B (38.4\%) and eight with CPT-A (61.5\%). Among the five patients with CPT-B, four were in group I, and one was in group II. Among the eight patients with CPT-A, six were in group I and two in group III.

\section{Analysis of potential SVR12 predictors}

Univariate analysis in patients treated with or without RBV indicated that absence of diabetes (OR, 4.7; 95\% CI, 1.4 to 16.0 ; $\mathrm{p}<0.01$ ), absence of HTA (OR, 4.6; 95\% CI, 1.3 to $15.4 ; \mathrm{p}<0.02$ ), and a disease classification of CPT-A (OR, 5.3; 95\% CI, 1.6 to $17.7 ; \mathrm{p}<0.01)$ were statistically significant predictors of SVR12. In contrast, antiviral regimen without RBV (OR, 0.17; 95\% CI 0.05 to $0.57 ; \mathrm{p}<0.003)$ was significantly associated with virologic failure (Table 4).

In the multivariate model, antiviral regimen without RBV (OR, 0.27 ; $95 \%$ CI, 0.07 to $1.0 ; \mathrm{p}<0.05$ ) and a classification of CPT-B or C (OR, 5.3; 95\% CI, 1.3 to $21.1 ; \mathrm{p}<0.01)$ remained significant negative predictors of SVR12 (Table 4).

Univariate analysis in group I patients versus group II indicated that absence of diabetes (OR, 4.3; 95\% CI, 1.1 to 16.7 ; $\mathrm{p}<0.03$ ), absence of HTA (OR, 4.6; 95\% CI, 1.19 to 18 ; $\mathrm{p}<0.02$ ) and a class CTP-A (OR, 5.9; 95\% CI, 1.63 to 21 ; $\mathrm{p}<0.007$ ) were significant predictor of SVR12. On the contrary, antiviral regi- 
Table 1. Demographic and Baseline Characteristics of Patients with G3-HCV Cirrhosis

\begin{tabular}{|c|c|c|c|c|}
\hline Characteristics & $\begin{array}{l}\text { Group I } \\
\text { DCV/SOF }\end{array}$ & $\begin{array}{c}\text { Group II } \\
\text { DCV/SOF/RBV flat dose }\end{array}$ & $\begin{array}{c}\text { Group III } \\
\text { DCV/SOF/ } \\
\text { RBV weight-based dose }\end{array}$ & $\mathrm{p}$-value \\
\hline Total no. & 70 & 87 & 76 & - \\
\hline Male sex & $56(80)$ & 73 (83.9) & $67(88.2)$ & 0.4 \\
\hline Age, yr & $53.6 \pm 8.4$ & $50.9 \pm 7.4$ & $52.3 \pm 7.1$ & 0.08 \\
\hline Race, white & 70 (100) & 87 (100) & $76(100)$ & 0.1 \\
\hline Risk factor & & & & 0.3 \\
\hline Previous or active PWID & $49(70)$ & $55(63.3)$ & $55(73.4)$ & \\
\hline Blood transfusion & $6(8.6)$ & $1(1.1)$ & $1(1.3)$ & \\
\hline Sexual behavior & 0 & 0 & $1(1.3)$ & \\
\hline Not determined & $15(21.4)$ & 31 (35.6) & $18(24)$ & \\
\hline Platelets <100/nL & $27(43.5)$ & $28(47.5)$ & $36(47.4)$ & 0.8 \\
\hline Hemoglobin, g/dL & $14(10-17)$ & $14.1(10-16.9)$ & $14.9(10-18)$ & 0.05 \\
\hline Total bilirubin, mg/dL & $1.0(0.3-3.3)$ & $1.1(0.3-3.2)$ & $0.9(0.3-3.3)$ & 0.1 \\
\hline Creatinine, mg/dL & $0.8(0.5-1.2)$ & $0.8(0.4-1.2)$ & $0.8(0.5-1.2)$ & 0.1 \\
\hline INR & $1.02(1-1.44)$ & $1.1(0.9-2.0)$ & $1.11(0.9-1.6)$ & 0.6 \\
\hline HCV RNA, >800,000 IU/mL & $20(28.5)^{*}$ & $41(51.2)^{*}$ & $31(44.2)$ & 0.02 \\
\hline Body mass index, $\mathrm{kg} / \mathrm{m}^{2}$ & & & & 0.2 \\
\hline$<25$ & $36(52.2)$ & $48(55)$ & $33(43.4)$ & \\
\hline $25-30$ & $21(30.4)$ & $25(29)$ & $29(38.2)$ & \\
\hline$>30$ & $13(17.4)$ & $14(16)$ & $14(18.4)$ & \\
\hline Diabetes & $11(15.7)$ & $10(12)$ & $10(13.3)$ & 0.4 \\
\hline Arterial hypertension & $13(18.5)$ & $9(10.7)$ & $12(16)$ & 0.3 \\
\hline Hypercholesterolemia & $7(10.1)$ & $1(1.2)$ & $1(1.3)$ & 0.006 \\
\hline Cryoglobulinemia & $3(4.3)$ & $5(6.0)$ & $10(13.3)$ & 0.1 \\
\hline Non-Hodgkin lymphoma & 0 & $1(1.2)$ & 0 & 0.7 \\
\hline Previous HCC & $6(8.7)$ & $3(3.5)$ & $4(5.3)$ & 0.3 \\
\hline Transient elastography, kPa & $21.3 \pm 10$ & $22.1 \pm 13$ & $22 \pm 14$ & 0.7 \\
\hline Child-Pugh-Turcotte & & & & $0.04^{*}$ \\
\hline A & $56(80)$ & 78 (89.7) & $71(93.4)$ & \\
\hline B & $14(20)^{*}$ & $8(9.2)$ & $5(6.6)^{*}$ & \\
\hline $\mathrm{C}$ & 0 & $1(1.1)$ & 0 & \\
\hline Esophageal varices size & & & & 0.1 \\
\hline None & $32(69.6)$ & $27(69.2)$ & $28(53.8)$ & \\
\hline $\mathrm{F} 1 \& \mathrm{~F} 2$ & $12(26.1)$ & $10(30.8)$ & $21(40.4)$ & \\
\hline F3 & $2(4.3)$ & $0(0)$ & $3(5.8)$ & \\
\hline Treatment-naïve & $47(67.1)^{*}$ & $33(37.9)^{*}$ & $44(57.9)$ & $0.001^{*}$ \\
\hline PegIFN/RBV & & & & $0.001^{*}$ \\
\hline Experienced & $16(30)^{*}$ & $51(58.6)^{*}$ & $29(38.2)$ & \\
\hline Relapse & $14(20)$ & $22(25.3)$ & $16(21.1)$ & \\
\hline No response & $7(10)$ & 29 (33.3) & $13(17.1)$ & \\
\hline PegIFN/RBV/TPV & & & & 0.5 \\
\hline Experienced & $1(1.4)$ & $2(2.3)$ & $2(2.6)$ & \\
\hline Relapse & $1(1.4)$ & $2(2.3)$ & $2(2.6)$ & \\
\hline Total SOF/RBV & & & & 0.7 \\
\hline Experienced & $1(1.4)$ & $1(1.1)$ & $1(1.3)$ & \\
\hline Relapse & 1 (1.4) & 1 (1.1) & 1 (1.3) & \\
\hline
\end{tabular}

Data are presented as number (\%), mean \pm SD, or median (range).

G3-HCV, genotype 3 hepatitis C virus; DCV, daclatasvir; SOF, sofosbuvir; RBV, ribavirin; PWID, people who inject drugs; INR, international normalized ratio for prothrombin time; HCC, hepatocellular carcinoma; PegIFN, pegylated interferon; TPV, telaprevir.

*Statistical significance between the two indicated groups. 
Table 2. Treatment Efficacy Outcomes and Reasons for Nonresponse in Patients with G3-HCV

\begin{tabular}{lcccc}
\hline \multicolumn{1}{c}{ Parameter } & Group I (n=70) & Group II (n=87) & Group III (n=76) & p-value \\
\hline HCV RNA undetectable at 24 weeks & $69(98.6)$ & $87(100)$ & $76(100)$ & NS \\
SVR4 & $63(90.0)$ & $85(97.7)$ & $74(97.4)$ & 0.04 \\
SVR12 & $61(87.1)$ & $85(97.7)$ & $74(97.3)$ & 0.007 \\
No SVR12 & $9(12.9)$ & $2(2.2)$ & $2(2.6)$ & 0.007 \\
Relapse & $8(88.9)$ & $2(2.2)$ & $2(2.6)$ & - \\
No response & $1(11.1)$ & 0 & 0 & - \\
Drop out & 0 & $1(1.1)$ & 0 & - \\
\hline
\end{tabular}

Data are presented as number (\%). Group I, daclatasvir (DCV) + sofosbuvir (SOF) without ribavirin (RBV); group II, DCV/SOF with RBV flat dose of $800 \mathrm{mg} / \mathrm{dL}$; group III, DCV/SOF with RBV weight-based dose.

G3-HCV, genotype 3 hepatitis C virus; NS, not significant; SVR, sustained viral response.

Table 3. Efficacy and Safety in CPT A versus CPT B/C Patients According to RBV Protocol

\begin{tabular}{|c|c|c|c|}
\hline Variable & CPT A without RBV n 56 & CPT B/C without RBV n 14 & $\mathrm{p}$-value \\
\hline HCV RNA SVR12 & 50 (89.3) & 11 (78.6) & 0.2 \\
\hline Anemia & $2(3.6)$ & $1(7.1)$ & 0.4 \\
\hline Fatigue & $5(8.9)$ & $5(35.7)$ & 0.02 \\
\hline Headache & 0 & 0 & NS \\
\hline Diarrhea & 0 & 0 & NS \\
\hline \multirow[t]{2}{*}{ SAE } & 0 & $1(7.1)$ & 0.2 \\
\hline & CPT A flat RBV nº 78 & $\mathrm{CPT} \mathrm{B} / \mathrm{C}$ flat $\mathrm{RBV} n^{\circ} 9$ & \\
\hline HCV RNA SVR12 & $78(100)$ & 7 (77.8) & 0.01 \\
\hline Anemia & $14(17.9)$ & $3(33.3)$ & 0.2 \\
\hline Fatigue & $17(21.8)$ & $2(22.2)$ & 0.6 \\
\hline Headache & $5(6.4)$ & 0 & 0.5 \\
\hline Diarrhea & $2(2.6)$ & 0 & 0.8 \\
\hline \multirow[t]{2}{*}{ SAE } & $1(1.3)$ & $1(11.1)$ & 0.08 \\
\hline & CPT A weight dose $\mathrm{RBV} \mathrm{n}^{\circ} 73$ & CPT B/C weight dose $\mathrm{RBV} n^{\circ} 5$ & \\
\hline HCV RNA SVR12 & $71(98)$ & $5(100)$ & 0.8 \\
\hline Anemia & $26(35.6)$ & $4(80)$ & 0.05 \\
\hline Fatigue & $25(34.2)$ & $3(60)$ & 0.2 \\
\hline Headache & $5(6.8)$ & $1(20)$ & 0.3 \\
\hline Diarrhea & $2(2.7)$ & 0 & 0.8 \\
\hline SAE & 0 & 0 & NS \\
\hline
\end{tabular}

Data are presented as number (\%).

CPT, Child-Pugh-Turcotte; RBV, ribavirin; HCV, hepatitis C virus; SAE, severe adverse event; NS, significant.

men not containing RBV was associated with virological failure $(\mathrm{OR}, 0.1 ; 95 \% \mathrm{CI}, 0.34$ to $0.7 ; \mathrm{p}<0.02)$. In the multivariate model a regimen with or without RBV did not influence SVR12, while a CPT-A was associated with a better SVR12 (Table 5).

Univariate analysis in group I patients versus group III indicated that absence of diabetes (OR, 4.1; 95\% CI, 1.0 to 15.7; $\mathrm{p}<0.03$ ), absence of HTA (OR, 4.6; 95\% CI, 0.99 to 13; $\mathrm{p}<0.05$ ) and no prior antiviral treatment were significant predictors of SVR12 (OR, 3.15; 95\% CI, 0.95 to 11 ; p<0.05). On the contrary, antiviral regimen without RBV was associated with virological failure (OR, 0.17; 95\% CI, 0.35 to 0.85 ; $<<0.03$ ). In the multivariate model a regimen without RBV was associated with virological failure (OR, $0.18 ; 95 \% \mathrm{CI}, 0.03$ to 0.18 ; $\mathrm{p}<0.04)$ while no prior antiviral treatment was a predictor of SVR12 (OR, 5.3; 95\% CI, 1.2 to 23; $\mathrm{p}<0.02$ ) (Table 5). 
Table 4. Univariate and Multivariate Analysis of Factors Associated with SVR at 12 Weeks in Patients with G3-HCV Cirrhosis with or without RBV

\begin{tabular}{|c|c|c|c|c|c|}
\hline \multirow{2}{*}{ Variable } & \multicolumn{3}{|c|}{ Univariable model } & \multicolumn{2}{|c|}{ Multivariable model } \\
\hline & SVR12 (\%) & OR $(95 \% \mathrm{CI})$ & $\mathrm{p}$-value & OR $(95 \%$ CI $)$ & $\mathrm{p}$-value \\
\hline Female vs male & 94.6 vs 94.4 & $1.0(0.2-4.8)$ & 0.6 & - & - \\
\hline Diabetes (no vs yes) & 96.4 vs 85.3 & $4.7(1.4-16.0)$ & 0.01 & $2.0(0.4-9.8)$ & 0.3 \\
\hline Arterial hypertension (no vs yes) & 96.4 vs 87.5 & $4.6(1.3-15.4)$ & 0.02 & $3.6(0.73-18)$ & 0.1 \\
\hline Cryoglobulinemia (no vs yes) & 94.8 vs 94.4 & $1.0(0.13-9.3)$ & 0.6 & - & - \\
\hline BMI $\left(\leq 25 \mathrm{~kg} / \mathrm{m}^{2}\right.$ vs $\left.>25 \mathrm{~kg} / \mathrm{m}^{2}\right)$ & 94.0 vs 95.6 & $0.72(0.2-2.3)$ & 0.4 & - & - \\
\hline PLT $\left(\leq 100 \times 10^{3} / \mu \mathrm{L}\right.$ vs $\left.>100 \times 10^{3} / \mu \mathrm{L}\right)$ & 95.3 vs 94.5 & $1.1(0.3-4.1)$ & 0.5 & - & - \\
\hline $\begin{array}{l}\text { HCV RNA }(\leq 800,000 \mathrm{IU} / \mathrm{mL} \text { vs } \\
>800,000 \mathrm{IU} / \mathrm{mL})\end{array}$ & 93.5 vs 95.7 & $0.65(0.19-2.2)$ & 0.3 & - & - \\
\hline CPT (A vs B/C) & 96.1 vs 82.1 & $5.3(1.6-17.7)$ & 0.01 & $5.3(1.3-21.1)$ & 0.01 \\
\hline Esophageal varices (no vs yes) & 94.3 vs 98.0 & $0.33(0.03-2.9)$ & 0.2 & - & - \\
\hline Naïve vs experienced & 96.0 vs 92.7 & $1.88(0.59-5.9)$ & 0.2 & - & - \\
\hline No RBV vs RBV & 87.1 vs 97.5 & $0.17(0.05-0.57)$ & 0.003 & $0.27(0.07-1.0)$ & 0.05 \\
\hline
\end{tabular}

SVR, sustained viral response; G3-HCV, genotype 3 hepatitis C virus; RBV, ribavirin; OR, odds ratio; CI, confidence interval; BMI, body mass index; PLT, platelets; CPT, Child-Pugh-Turcotte.

Table 5. Multivariate Analysis of Factors Associated with SVR12

\begin{tabular}{lcccc}
\hline & \multicolumn{2}{c}{ Group I vs group II } & \multicolumn{2}{c}{ Group I vs group III } \\
\cline { 2 - 3 } \cline { 5 - 5 } & OR (95\% CI) & p-value & OR (95\% CI) & p-value \\
\hline CPT (A vs B) & $6.8(1.5-30)$ & 0.01 & - & - \\
HTA (no vs yes) & $3.86(0.5-27)$ & 0.1 & $2.0(0.33-12)$ & 0.4 \\
RBV (no vs yes) & $0.2(0.55-27)$ & 0.1 & $0.18(0.03-0.98)$ & 0.02 \\
Diabetes (no vs yes) & - & - & $2.6(0.41-16)$ & 0.3 \\
Nä̈ve (yes vs no) & - & - & $5.3(1.2-23)$ & 0.02
\end{tabular}

Group I, daclatasvir (DCV) + sofosbuvir (SOF) without ribavirin (RBV); group II, DCV/SOF with RBV flat dose of $800 \mathrm{mg} / \mathrm{dL}$; group III, DCV/SOF with RBV weight-based dose.

SVR, sustained viral response; SVR12, SVR at 12 weeks; OR, odds ratio; CI, confidence interval; CPT, Child-Pugh-Turcotte; HTA, hypertension.

Table 6. Characteristics of Patients with G3-HCV Cirrhosis Who Showed no Response to Past and Current Antiviral Treatments

\begin{tabular}{cclcccc}
\hline Patient & $\begin{array}{c}\text { Age, yr } \\
\text { sex }\end{array}$ & \multicolumn{1}{c}{ Past treatment } & $\begin{array}{c}\text { HCV RNA } \\
>800,000 \text { IU/mL }\end{array}$ & CPT class & Treatment & $\begin{array}{c}\text { NS5A/NS5B resistance test, } \\
\text { posttreatment }\end{array}$ \\
\hline 1 & $50 / \mathrm{M}$ & Relapsed after PegIFN/RBV & No & A & DCV/SOF/RBV 1,000 mg & Not performed \\
2 & $56 / \mathrm{F}$ & None & Yes & A & DCV/SOF & Y93H \\
3 & $52 / \mathrm{M}$ & Relapsed after PegIFN/RBV & No & A & DCV/SOF & Y93H \\
4 & $50 / \mathrm{M}$ & None & No & B & DCV/SOF & Y93H \\
5 & $60 / \mathrm{M}$ & No response to PegIFN/RBV & Yes & A & DCV/SOF & Y93H \\
6 & $52 / \mathrm{M}$ & No response to PegIFN/RBV & No & A & DCV/SOF/RBV 1,000 mg & Y93H-L159F- L31M \\
7 & $52 / \mathrm{M}$ & Relapsed after SOF/RBV & No & A & DCV/SOF & Y93H \\
8 & $48 / \mathrm{F}$ & None & No & B & DCV/SOF & Y93H \\
9 & $46 / \mathrm{M}$ & None & Yes & B & DCV/SOF/RBV 800 mg & Y93H \\
10 & $41 / \mathrm{M}$ & None & No & B & DCV/SOF & Y93H \\
11 & $57 / M$ & No response to PegIFN/RBV & Yes & A & DCV/SOF & Y93H \\
12 & $61 / \mathrm{M}$ & Relapsed after PegIFN/RBV & Yes & A & DCV/SOF & None \\
\hline
\end{tabular}

G3-HCV, genotype 3 hepatitis C virus; CPT, Child-Pugh-Turcotte; M, male; F, female; PegIFN, pegylated interferon; RBV, ribavirin; DCV, daclatasvir; SOF, sofosbuvir. 


\section{Virological resistance}

Overall, 12 patients did not respond to antiviral treatment. Eight patients of group I had a relapse after completing the treatment, and one patient had a breakthrough. Four were treatment-naïve (44.4\%), two had relapsed after a previous treatment with PegIFN/RBV (22.2\%), two showed no response to PegIFN/RBV (22.2\%), and one had relapsed after SOF/RBV (11.1\%). The patient that had a breakthrough, had previously relapsed after a treatment with SOF/RBV. Two patients of group II had a relapse after the completion of treatment. One patient was treatment-naïve and one patient had relapsed after a previous treatment of SOF/RBV. Two patients in group III relapsed after treatment; one patient had previously shown no response to PegIFN/RBV and one had relapsed after a previous treatment with PegIFN/RBV. One patient dropped out, due to a recurrence of HCC, during the sixth month of treatment. Antiviral failure occurred more frequently in patients of group I compared to group II $(12.9 \%$ vs $2.3 \%, \mathrm{p}<0.009)$ and group III (13\% vs 2.6, $\mathrm{p}<0.01)$. No baseline test was performed for RAVs. In 11 out of 12 patients that failed DCV/SOF with or without RBV, a resistance test was performed for the NS5A and NS5B proteins at the time of failure. At failure, one patient in group III with no response to a previous PegIFN/RBV treatment showed a NS5B RAV, L159F, which was associated with NS5A RAV Y93H, L31M. In another patient in group III with no response to a previous PegIFN/RBV treatment, no re- sistance test was performed for the NS5A/NS5B proteins. The isolated RAV Y93H was identified in eight patients of group I, and in one patient of group II. In one patient of group I that had previously relapsed after Peg/RBV, no resistance to NS5A/NS5B was found at failure (Table 6).

\section{Safety}

Grades 1 and 2 anemia were more frequent in group II (19.5\%) and III (39.5\%) compared to group I (4.2\%, p<0.0001). A higher incidence of grades 1 and 2 anemia was found in group III compared to group II (OR, 2.6; 95\% CI, 1.26 to 5.76; $\mathrm{p}<0.005)$. No case of grade 3 or 4 anemia that required a blood transfusion was reported in the three groups of patients. Reduction of the RBV daily dose was reported for 12 patients. RBV was reduced more frequently in group III (14\%) than in group II (2.3\%: OR, 4.74; 95\% CI, 0.97 to 32 ; $\mathrm{p}<0.032)$. In all patients, a reduction in the RBV daily dose resulted in an increase in hemoglobin levels. Fatigue was observed in 10 patients in group I (14.3\%), 19 in group II (21.8\%), and 28 in group III $(36.8 \%, p<0.005)$. Fatigue occurred significantly more frequently in group III than in group II (OR, 2.0; 95\% CI, 0.99 to $4.41 ; \mathrm{p}<0.03)$. Higher incidences of grades 1 and 2 anemia and fatigue were reported in patients with CPTB compared to those with CPT-A, but the difference was not statistically significant $(29.6 \%$ vs $20.4 \%$ and $37 \%$ vs $22.9 \%$ respectively). AEs occurred more frequently in group III than in group II (86.8\% vs 49.4\%: OR, 6.7; 95\% CI, 2.9 to 16 ;

Table 7. Safety and Tolerability of Treatment Regimens in Patients with G3-HCV Cirrhosis

\begin{tabular}{|c|c|c|c|c|}
\hline Parameter & Group I (n=70) & Group II (n=87) & Group III ( $\mathrm{n}=76)$ & p-value \\
\hline Any AE & $13(14.2)$ & $43(49.4)$ & $66(86.8)$ & - \\
\hline Death & 0 & $1(1.1)$ & 0 & - \\
\hline SAEs & $1(1.6)$ & $2(2.2)$ & 0 & 0.4 \\
\hline Recurrence ascites & $1(1.6)$ & 0 & 0 & \\
\hline Pneumonia & 0 & $1(1.1)$ & 0 & \\
\hline Atrial fibrillation & 0 & $1(1.1)$ & 0 & \\
\hline $\mathrm{AE}$ that led to discontinuation & 0 & 0 & 0 & - \\
\hline \multicolumn{5}{|l|}{ AEs } \\
\hline Fatigue & $10(14.3)$ & $19(21.8)$ & $28(36.8)$ & 0.005 \\
\hline Anemia (grade 2/3) & $3(4.8)$ & $17(19.5)$ & $30(39.5)$ & 0.000 \\
\hline Headache & 0 & $5(5.7)$ & $6(7.9)$ & 0.08 \\
\hline Diarrhea & 0 & $2(2.3)$ & $2(2.6)$ & 0.4 \\
\hline Drop out & 0 & $1(1.1)$ & 0 & - \\
\hline RBV dose reduction & NA & $2(2.3)$ & $10(14)$ & 0.007 \\
\hline HCC de novo & $1(1.4)^{*}$ & 0 & 0 & - \\
\hline HCC recurrence & 0 & $1(1.1)^{\dagger}$ & 0 & - \\
\hline
\end{tabular}

Data are presented as number (\%). Group I, daclatasvir (DCV) + sofosbuvir (SOF) without ribavirin (RBV); group II, DCV/SOF with RBV flat dose of $800 \mathrm{mg} / \mathrm{dL}$; group III, DCV/SOF with RBV weight-based dose.

G3-HCV, genotype 3 hepatitis C virus; AE, adverse event; SAE, severe adverse event; NA, not applicable; HCC, hepatocellular carcinoma.

*At 8 months after the end of treatment; ${ }^{\dagger}$ Dropped out due to HCC recurrence. 
$\mathrm{p}<0.001)$. No patient discontinued antiviral therapy due to AEs.

One patient in group I and two patients in group II experienced SAEs that required hospitalization (respectively, one recurrence of ascites, one pneumonia case, and one atrial fibrillation case) (Table 7). No patient in either group discontinued antiviral treatment due to SAEs.

\section{Hepatocellular carcinoma}

HCC was previously diagnosed in 13 out of 233 patients (5.6\%). In group I, six out of 70 patients had previous HCC (8.7\%) compared to three out of $87(3.5 \%)$ in group II and four out of 75 (5.3\%) in group III (no significant differences). After a follow up of $10 \pm 5$ months, de novo HCC was identified in one patient with CPT-A in group I and HCC recurrence was reported in one patient with CPT-B in group II. The latter patient dropped out of the study after negativization of $\mathrm{HCV}$ RNA at 24 weeks of treatment. No recurrence of HCC was noted in other patients with previous HCC after a follow-up of $10 \pm 5$ months.

\section{DISCUSSION}

Although current treatment regimens for different HCV genotypes are effective in most scenarios, SVR rates tend to be lower among patients with G3-HCV and advanced liver disease. G3 is commonly associated with steatosis, progression to cirrhosis, and the development of HCC. ${ }^{1,2}$ Overall, the available data from registry and real-world studies have suggested that patients with G3-HCV cirrhosis are more difficult to cure than patients with other HCV genotypes. ${ }^{3-5}$ It is critical to improve the SVR in these patients, because achieving an undetectable viral load is associated with decreased hepatic morbidity and mortality. Previous antiviral DAA regimens with SOF/RBV showed low SVR12 achievement in patients with G3-HCV cirrhosis, whether they were treatment-naïve or treatment-experienced. Zeuzem et al. ${ }^{6}$ demonstrated that patients treated with SOF/RBV weight-based doses for 24 weeks had a low SVR12 (62\%). Similarly, Feld et al. ${ }^{7}$ showed low SVR12 rates in treatment-naïve and treatment-experienced patients with cirrhosis that received the same antiviral regimen (44.8\%). Paradoxically, treatments based on PegIFN/RBV achieved higher SVR12 rates, but also higher AEs compared to treatments based on SOF/RBV. ${ }^{8}$ Association of SOF/RBV is not further recommended in cirrhotic G3 patients. Consequently, other antiviral combinations were attempted with SOF and DCV. The Ally-3 trial analyzed a group of patients with G3-HCV cirrhosis treated with SOF/DCV without RBV for 12 weeks; they found low SVR12 rates (58\% in treatmentnaïve and 69\% in experienced groups). ${ }^{5}$ In the Ally-3+ study, Leroy et al. ${ }^{3}$ analyzed how RBV added to a regimen of DCV/ SOF for a duration of 12 or 16 weeks increased SVR12 in patients with G3-HCV cirrhosis. They demonstrated higher SVR12 rates compared to those of the Ally-3 study, with SVR12 of $86 \%$ and $88 \%$ in patients treated for 12 and 16 weeks, respectively. Although the small sample size of patients with G3-HCV cirrhosis prevented any solid conclusions regarding the role of RBV, those findings indicated that RBV provided important added value in this "difficult-to-treat population." However, it remains difficult to determine the optimal treatment duration for SOF/DCV with or without RBV for patients with G3-HCV. In addressing this question, different studies have shown that increasing the duration of therapy from 12 to 24 weeks was associated with a better SVR. ${ }^{8,10,11}$ In a study by Herzer et al., ${ }^{11} 126$ patients with advanced liver fibrosis were analyzed; they received DCV/SOF with or without a weight-based RBV dose for 24 weeks. They found SVR12 of 89\% with DCV/SOF (41/46 patients) and 88\% (29/33) for $\mathrm{DCV} / \mathrm{SOF} / \mathrm{RBV}$. Those authors concluded that response rates were similar in the two treatment groups, and they suggested that RBV did not confer an efficacy benefit to the DCV/SOF regimen, even when treatment was extended to 24 weeks. Similarly, Hézode et al. ${ }^{12}$ studied patients with compensated and decompensated cirrhosis treated for 24 weeks with DCV/ SOF or DCV/SOF/RBV (weight-based). They showed SVR12 of $86 \%$ and $82 \%$, respectively. In that study, it is important to consider that, at baseline, more than 50\% of patients treated with DCV/SOF/RBV had a Model for End-Stage Liver Disease category $>10$, a lower number of platelets, and lower albumin levels, compared to patients treated without RBV. These differences in patient characteristics indicated a more advanced liver disease in the group treated with RBV compared to those treated without RBV, which might explain the difference in SVR12. On the other hand, Alonso et al. ${ }^{10}$ conducted a multicenter observational study with 94 patients with G3-HCV that had compensated and decompensated cirrhosis and were treated with $\mathrm{DCV} / \mathrm{SOF} \pm \mathrm{RBV}$ weight-based dose regimens for 24 weeks. They found SVR12 rates of 96\% in patients treated with RBV and 90\% in patients treated without RBV. Similarly, Cornberg et al. ${ }^{8}$ showed that patients with G3-HCV cirrhosis that were either treatment-naïve or treatment-experienced achieved excellent SVR12 in 24-week treatments with a DCV/ SOF/RBV weight-based regimen (98\%) compared to patients treated with DCV/SOF (87\%). These conflicting findings may result from different patient selection-biases in clinical registered trials and real world experiences.

Other DAA regimens were also employed to treat patients with G3-HCV cirrhosis. Curry and Charlton ${ }^{13}$ tested three different regimens SOF/velpatasvir (VEL) for 12 weeks, SOF/ VEL/RBV for 12 weeks, and SOF/VEL for 24 weeks, in patients with G3-HCV with decompensated cirrhosis. The SVR rates were, respectively, 50\%, 85\%, and 50\%. Thus, in that case, RBV also played an important role in enhancing the SVR in this "difficult-to-treat population." In an interesting 
study by Pianko et al., ${ }^{14} 52$ patients with G3-HCV cirrhosis were screened with a pretreatment NS5A resistance test. Patients were then treated with SOF/VEL/RBV or SOF/VEL without RBV for 12 weeks. They demonstrated a SRV12 of 95\%, irrespective of the presence of RBV, in patients without NS5A RAVs. In contrast, a significant SVR reduction (respectively of $75 \%$ vs $100 \%$ ) was observed in patients with NS5A RAVs at baseline treated with regimen without RBV compared to patients treated with a regimen containing RBV, showing a penalizing effect of RBV-free regimes in those with NS5A RAVs at baseline. ${ }^{13,14}$ That study stressed the importance of RBV as additional antiviral component in patients with baseline RAVs. However, it is not possible to perform a NS5A RAV test routinely at baseline in all centers that provide DAA treatments. Therefore, it might be wise to include RBV in the DAA therapy whenever there is a suspicion that patients are at high risk of harboring RAVs (i.e., treatment-experienced patients). In our study, NS5A RAV screening was not performed at baseline, and due to the study design, we did not assign the administration of RBV based on the relative risk of RAVs. In particular, in the group of patients treated with DCV/SOF without RBV, we found a high incidence of the Y93H mutation among patients that had relapsed. This mutation might explain the low efficiency of this antiviral regimen. Moreover, we speculate that suboptimal antiviral treatment might allow selective survival of RAVs, and thus, fail to provide antiviral activity. At present, European Association Study Liver guidelines recommend an association of glecaprevir/pibrentasvir for 12 or 16 weeks but only in compensated naïve or experienced G3 cirrhosis. ${ }^{15}$ This regimen showed a SVR12 of 98\% and 96\% respectively in naïve and experienced compensated cirrhotic patients treated for 12 and 16 weeks. ${ }^{16}$ But this DAA association is not indicate in decompensated cirrhosis or in cirrhotic with Model for End-Stage Liver Disease score $>10$. Similarly, SOF/VEL did not show an optimal SVR12 in G3 naïve or experienced cirrhotic patients as reported above.

Although the limit of our study is that is not a randomized study, added RBV was homogenously distributed across the 12 centers and we are aware that in group I a higher percentage of CPT-B patients treated without RBV was present compared to other groups. We noted in CPT-B patients treated with weight dose RBV an higher this could explain why between group I and II a regimen with or without RBV did not influence SVR12, while a CPT-A was associated to a better SVR12.

Reig et al. ${ }^{17}$ observed in patients treated with DAAs a high rate of HCC recurrence (27.6\%) in patients with previous HCC complete radiological response; in our experience a lower recurrence rate of HCC at 6 months (7.7\%) was disclosed. This result $(9.2 \%)$ is similar to that reported by Cabibbo et al. ${ }^{18}$ In our study a correct evaluation by CT or MRI of all the patients previous treated for HCC to exclude posttreatment residual HCC was employed. As suggested from other authors, long term observational studies will be needed to determine the effective risk for developing HCC recurrence or occurrence in patients treated with DAAs. ${ }^{19}$

In conclusion, this study investigated the role of RBV in achieving SVR12 in a large multicenter cohort of 233 patients with G3-HCV cirrhosis, with and without compensation. We found that patients treated without RBV achieved a lower SVR12 compared to patients treated with either weight-based RBV or a flat RBV dose. Of interest, although high SVR12 rates were achieved with DCV/SOF/RBV for 24 weeks, irrespective of how the RBV was dosed, patients treated with the DCV/SOF/ RBV flat dose (800 mg/day) had significantly fewer AEs than patients treated with the DCV/SOF/RBV weight-based dose. Thus, RBV intolerance was lower with the flat dose than with the weight-based dose. We concluded that, in this "difficult-totreat population," the antiviral regimen of DCV/SOF/RBV flat dose (800 mg/day) was an effective regimen, in terms of high SVR12 rates and low AE incidence. In CPT-B patients, antiviral regimen of $\mathrm{DCV} / \mathrm{SOF} / \mathrm{RBV}$ weight dose showed higher antivira efficacy compared to other groups but this regimen is burdened by more AEs.

When and how to use RBV with DCV/SOF regimens has become the holy grail for investigators interested in eradicating G3-HCV infections. The advent of new DAAs has reduced the role of RBV to a marginal companion in antiviral treatment regimens. However, in the present study, we have shown that RBV remains an important treatment component for increasing SVR12 in the special population of patients with G3-HCV and in particular in decompensated cirrhosis and in experienced patients with compensated cirrhosis. Thus, withholding RBV must be restricted to treatments for naïve compensated cirrhotic patients with a clear contraindication to RBV.

\section{CONFLICTS OF INTEREST}

No potential conflict of interest relevant to this article was reported.

\section{ACKNOWLEDGEMENTS}

Working Group CLEO. The following authors have contributed equally to the realization of the manuscript, in terms of casistics and review of the manuscript: Francesca Wanda Chiesara (Gastroenterology, San Filippo Neri Hospital, Rome, Italy), Barbara Imperatrice (Digestive and Liver Disease Unit, Sant'Andrea Hospital, Rome, Italy), Paola Begini (Digestive and Liver Disease Unit, Sant'Andrea Hospital, Rome, Italy), Giovanni Vennarecci (Division of General Surgery and Liver Transplantation, San Camillo Forlanini Hospital, Rome, Italy), Francesca Romana Ponziani (Internal Medicine, Catholic Uni- 
versity, Rome, Italy), Roberto Gulminetti (Infectious Disease, IRCCS San Matteo, Pavia, Italy), Rodolfo Sacco (Gastroenterology, Azienda Ospedaliera Pisana Pisa, Italy), Antonio Ascione (Center for Liver Disease, Fatebenefratelli Hospital, Napoli, Italy), Caterina Furlan (Infectious Disease University of Rome "La Sapienza" Rome, Italy), Roberto Villani (Liver and Transplant Unit, San Camillo Forlanini Hospital, Rome, Italy), Cecilia D’Ambrosio (Liver and Transplant Unit, San Camillo Forlanini Hospital, Rome, Italy).

\section{AUTHOR CONTRIBUTIONS}

Concept and design, data analysis, interpretation, critical revision and study supervision: A.P. Data acquisition and study supervision: V.M., V.G. Data acquisition and data analysis: M.D. Statistical analysis and technical material support: V.P.P. Statistical analysis and data analysis: P.V. Data interpretation: P.T. Drafting the manuscript: A.I., F.D.C., A.M.I. Data acquisition: A.M., S.D., G.C., G.V. Data acquisition and material support: S.B. Critical revision of manuscript: M.M. Critical revision of manuscript and data acquisition: G.S. Data analysis and interpretation: V.I., M.P., G.B. Data acquisition and technical support: L.F. Analysis interpretation and critical revision: G.M.E.

\section{ORCID}

Adriano Pellicell

Vincenzo Messina

Valerio Giannelli

Pascal Vignally

Sergio Babudieri

Massimo Marignani

Maurizio Pompili

Luca Fontanella

Giuseppe M Ettorre

Giovanni Vennarecci

Antonio Massimo Ippolito

https://orcid.org/0000-0003-2135-5209

Giorgio Barbarini

https://orcid.org/0000-0003-2712-0259

\section{REFERENCES}

1. Kawaguchi Y, Mizuta T. Interaction between hepatitis C virus and metabolic factors. World J Gastroenterol 2014;20:28882901.

2. Kanwal F, Kramer JR, Ilyas J, Duan Z, El-Serag HB. HCV genotype 3 is associated with an increased risk of cirrhosis and hepatocellular cancer in a national sample of U.S. Veterans with HCV. Hepatology 2014;60:98-105.

3. Leroy V, Angus P, Bronowicki JP, et al. Daclatasvir, sofosbuvir, and ribavirin for hepatitis $\mathrm{C}$ virus genotype 3 and advanced liver disease: a randomized phase III study (ALLY-3+). Hepatology 2016;63:1430-1441.

4. Lontok E, Mani N, Harrington PR, Miller V. Closing in on the target: sustained virologic response in hepatitis $\mathrm{C}$ virus genotype infection response-guided therapy. Clin Infect Dis 2013;56:14661470.

5. Nelson DR, Cooper JN, Lalezari JP, et al. All-oral 12-week treatment with daclatasvir plus sofosbuvir in patients with hepatitis $C$ virus genotype 3 infection: ALLY-3 phase III study. Hepatology 2015;61:1127-1135.

6. Zeuzem S, Dusheiko GM, Salupere R, et al. Sofosbuvir and ribavirin in HCV genotypes 2 and 3. N Engl J Med 2014;370:19932001.

7. Feld JJ, Maan R, Zeuzem S, et al. Effectiveness and safety of sofosbuvir-based regimens for chronic HCV genotype 3 infection: results of the HCV-TARGET study. Clin Infect Dis 2016;63:776783.

8. Cornberg M, Petersen J, Schober A, et al. Real-world use, effectiveness and safety of anti-viral treatment in chronic hepatitis $C$ genotype 3 infection. Aliment Pharmacol Ther 2017;45:688-700.

9. European Association for Study of Liver. EASL clinical practice guidelines: management of hepatitis $\mathrm{C}$ virus infection. J Hepatol 2014;60:392-420.

10. Alonso S, Riveiro-Barciela M, Fernandez I et al. Effectiveness and safety of sofosbuvir-based regimens plus an NS5A inhibitor for patients with HCV genotype 3 infection and cirrhosis: results of a multicenter real-life cohort. J Viral Hepat 2017;24:304-311.

11. Herzer K, Welzel TM, Spengler U, et al. Real-world experience with daclatasvir plus sofosbuvir \pm ribavirin for post-liver transplant HCV recurrence and severe liver disease. Transpl Int 2017;30:243255.

12. Hézode C, Lebray P, De Ledinghen V, et al. Daclatasvir plus sofosbuvir, with or without ribavirin, for hepatitis $\mathrm{C}$ virus genotype 3 in a French early access programme. Liver Int 2017;37:13141324.

13. Curry MP, Charlton M. Sofosbuvir and velpatasvir for patients with HCV infection. N Engl J Med 2016;374:1688.

14. Pianko S, Flamm SL, Shiffman ML, et al. Sofosbuvir plus velpatasvir combination therapy for treatment-experienced patients with genotype 1 or 3 hepatitis $\mathrm{C}$ virus infection: a randomized trial. Ann Intern Med 2015;163:809-817.

15. European Association for the Study of the Liver. EASL recommendations on treatment of hepatitis C 2018. J Hepatol 2018;69:461511.

16. Wyles D, Poordad F, Wang S, et al. Glecaprevir/pibrentasvir for hepatitis C virus genotype 3 patients with cirrhosis and/or prior treatment experience: a partially randomized phase 3 clinical trial. Hepatology 2018;67:514-523.

17. Reig M, Mariño Z, Perelló C, et al. Unexpected high rate of early tumor recurrence in patients with HCV-related HCC undergoing interferon-free therapy. J Hepatol 2016;65:719-726. 
18. Cabibbo G, Petta S, Calvaruso V, et al. Is early recurrence of hepatocellular carcinoma in HCV cirrhotic patients affected by treatment with direct-acting antivirals? A prospective multicentre study. Aliment Pharmacol Ther 2017;46:688-695.
19. Chinchilla-López P, Qi X, Yoshida EM, Méndez-Sánchez N. The direct-acting antivirals for hepatitis $\mathrm{C}$ virus and the risk for hepatocellular carcinoma. Ann Hepatol 2017;16:328-330. 